\title{
APOBEC1 complementation factor (A1CF) is dispensable for C-to-U RNA editing in vivo
}

\author{
ELIZABETH M. SNYDER, CHRISTOPHER MCCARTY, ADRIENNE MEHALOW, KAREN L. SVENSON, \\ STEPHEN A. MURRAY, RON KORSTANJE, and ROBERT E. BRAUN \\ The Jackson Laboratory, Bar Harbor, Maine 04609, USA
}

\begin{abstract}
Editing of the human and murine $A p o B$ mRNA by APOBEC1, the catalytic enzyme of the protein complex that catalyzes C-to-U RNA editing, creates an internal stop codon within the APOB coding sequence, generating two protein isoforms. It has been long held that APOBEC1-mediated editing activity is dependent on the RNA binding protein A1CF. The function of A1CF in adult tissues has not been reported because a previously reported null allele displays embryonic lethality. This work aimed to address the function of A1CF in adult mouse tissues using a conditional A1cf allele. Unexpectedly, A1cf-null mice were viable and fertile with modest defects in hematopoietic, immune, and metabolic parameters. C-to-U RNA editing was quantified for multiple targets, including $A p o B$, in the small intestine and liver. In all cases, no changes in RNA editing efficiency were observed. Blood plasma analysis demonstrated a male-specific increase in solute concentration and increased cellularity in the glomeruli of male A1cf-null mice. Urine analysis showed a reduction in solute concentration, suggesting abnormal water homeostasis and possible kidney abnormalities exclusive to the male. Computational identification of kidney C-to-U editing sites from polyadenylated RNA-sequencing identified a number of editing sites exclusive to the kidney. However, molecular analysis of kidney C-to-U editing showed no changes in editing efficiency with A1CF loss. Taken together, these observations demonstrate that A1CF does not act as the APOBEC1 complementation factor in vivo under normal physiological conditions and suggests new roles for $\mathrm{A} 1 \mathrm{CF}$, specifically within the male adult kidney.
\end{abstract}

Keywords: RNA editing; ACF; APOBEC1; APOB; kidney

\section{INTRODUCTION}

Mammalian RNA editing, the modification of a nucleotide within an intact RNA molecule, occurs in two distinct forms: adenosine to inosine (A-to-I) and cytosine to uracil (C-to$\mathrm{U})$. Both types of editing influence RNA function and its regulation. Deficiency in RNA editing can lead to substantial physiological defects including embryonic or postnatal lethality in the case of A-to-I editing (Higuchi et al. 2000; Hartner et al. 2004) and metabolic disorders in the case of C-to-U editing (Nakamuta et al. 1996). In mammals, the most common form of RNA editing is A-to-I, which is both relatively widespread throughout the body and impacts a broad range of targets. In contrast, C-to-U editing is highly tissue and target dependent. Although genetic regulation of A-to-I editing is primarily dependent on cis-acting factors within the target RNAs themselves, $\mathrm{C}$-to- $\mathrm{U}$ editing appears to be regulated by a limited number of trans-acting factors (Gu et al. 2016).

Corresponding author: bob.braun@jax.org

Article is online at http://www.rnajournal.org/cgi/doi/10.1261/rna.058818. 116. Freely available online through the RNA Open Access option.
The canonical C-to- $U$ editing event in mammals occurs in the apolipoprotein $\mathrm{B}$ mRNA and results in the production of two distinct proteins, the long form (ApoB-100) and the short form (ApoB-48), encoded by the same message. The short form is a product of a single C-to- $\mathrm{U}$ editing event that creates a stop codon upstream of the genome encoded stop (Powell et al. 1987). In humans, this editing event is observed exclusively in the small intestine while in rodents it occurs in the small intestine and, to a lesser degree, the liver (Teng et al. 1990).

The complex responsible for $A p o B$ editing requires both a catalytic and RNA binding component (Davies et al. 1989; Driscoll et al. 1989) and was initially identified based on the catalytic activity of the complex (Driscoll and Casanova 1990; Smith et al. 1991). Several years after the identification of the editing complex, the catalytic component, APOBEC1 (apolipoprotein B mRNA editing enzyme catalytic subunit 1), was cloned and shown to catalyze C-to-U editing in vitro (Teng et al. 1993). However, it was

(C) 2017 Snyder et al. This article, published in $R N A$, is available under a Creative Commons License (Attribution 4.0 International), as described at http://creativecommons.org/licenses/by/4.0/. 
evident the full editing complex required additional components aside from APOBEC1, which did not contain the necessary RNA sequence specificity to recognize the known substrate. Substrate recognition by the editing complex was further refined by the identification of an 11-nt mooring sequence within the target mRNA which, when mutated, partially or completely abrogated APOBEC1-mediated editing (Mehta and Driscoll 1998).

Lacking the necessary sequence-specific RNA binding motif, APOBEC1 must rely on a sequence-dependent RNA binding protein to confer its target specificity. In 2000, two groups identified the APOBEC1 complementation factor (ACF, recently renamed $\mathrm{A} 1 \mathrm{CF}$ ) as the likely $\mathrm{APOBEC} 1$ cofactor (Lellek et al. 2000; Mehta et al. 2000). Recombinant A1CF protein was shown to complement APOBEC1 in vitro editing activity and was dependent upon the $A p o B$ mooring sequences (Mehta et al. 2000). Over the course of several years, many groups demonstrated the ability of A1CF to complement APOBEC1's editing capacity in vitro, relying on purified recombinant proteins (Mehta and Driscoll 2002; Chester et al. 2004) or heterologous expression systems (Chester et al. 2003; Sowden et al. 2004; Severi and Conticello 2015).

Unlike $A p o B$ editing, relevant levels of which are limited to the small intestine and liver, APOBEC1 is detectable in a much wider range of tissues (Teng et al. 1993; Nakamuta et al. 1995), suggesting APOBEC1's function may not be limited to the regulation of C-to-U editing. However, while global ablation of Apobec1 in a mouse model resulted in a complete loss of $A p o B$ editing, there were no gross impacts on fecundity or fertility (Hirano et al. 1996) and only moderate impacts on serum lipoprotein profiles (Nakamuta et al. 1996). In contrast, while A1CF and APOBEC1 have very similar expression profiles in adult tissues, there was strong evidence that A1CF may have roles outside of C-toU editing (Sowden et al. 2002; Chester et al. 2003). This hypothesis gained even more credence when a global knockout of $A 1 c f\left(A 1 c f^{t m 1 D d s n}\right)$ was reported to display early embryonic lethality (Blanc et al. 2005), a phenotype substantially more severe than the Apobec1 knockout mice. Given the apparent role of A1CF in early embryogenesis, it has been difficult to assess its role in adult tissues, specifically the liver and small intestine. To test the role of A1cf in adult liver and small intestine RNA editing, we utilized mice carrying a conditional allele of Alcf.

\section{RESULTS}

\section{Global loss of A1CF does not result in embryonic lethality}

A knockout-first conditional allele of $A 1 c f\left(A 1 c f^{f m 1 a(E U C O M M)}\right.$ Hmgu ) was generated by the Knockout Mouse Program (KOMP) at The Jackson Laboratory. Global Alcf deletion $\left(A 1 c f^{t m l b}\right)$ was produced by excision of the neomycin selection marker and its floxed fourth exon via Sox2-driven
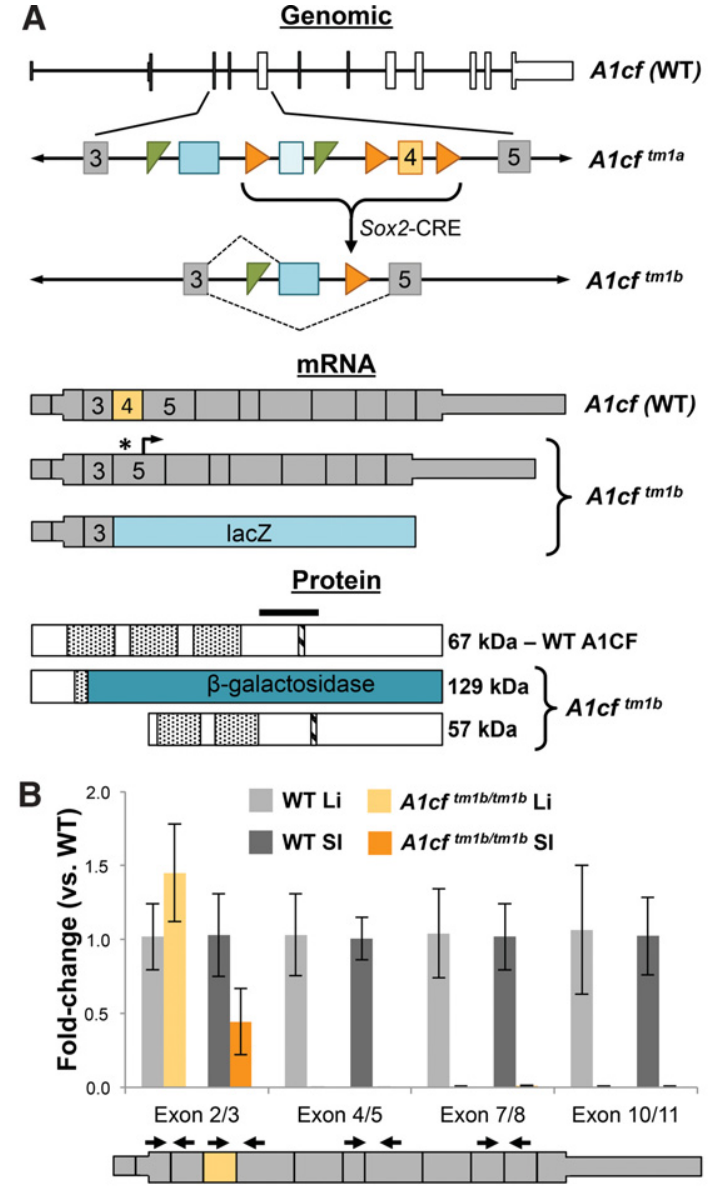

C

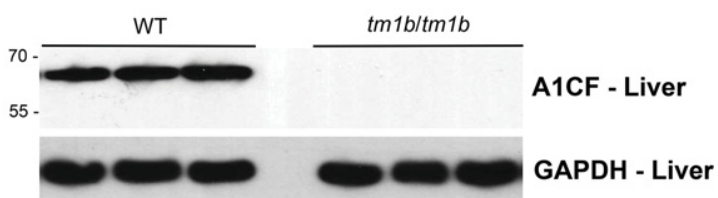

FIGURE 1. The $A 1 c f^{t m 1 b}$ allele is a true A1CF-null allele. (A) Schematic of genomic, RNA, and protein impacts of the $A 1 c f^{t m 1 b}$ allele. Genomic schematic of the WT Alcf allele with derivation and structure of $A 1 c f^{t m 1 b}$. (Gray and orange boxes) Alcf exons; (numbers) exon position within the Alcf locus; (blue boxes) lacZ (dark) and neo (light) cassettes; (green triangles) FRT sites; (orange triangles) loxP sites; (dashed lines) potential splicing events from the mutant allele. The $A 1 c f^{\mathrm{fm} 1 b}$ allele may produce multiple mRNAs, one including the generation of a premature stop codon $\left(^{*}\right)$ upstream of a new $3^{\prime}$ open reading frame (arrow), and another encoding a chimeric transcript of $A 1 c f$ and lacZ. (Thick boxes) Open reading frame. (Orange box) tm $1 b$ targeted exon. Schematic of WT and putative mutant AlCF proteins generated from the Alcf ${ }^{t m 1 b}$ allele. (Stippled boxes) RNA recognition motifs; (hatched box) nuclear localization signal; (black bar) antigen site for anti-AlCF antibody. (B) qRT-PCR analysis of the A1cf mRNA in WT and tm $1 b$ liver and small intestine. Exon junctions assessed indicated in graph and on WT mRNA schematic below ( $n \geq 4$, error, standard deviation). (C) Western blot detection of A1CF in liver with GAPDH as a representative loading control ( $n=3$ per genotype). Molecular weight in $\mathrm{kDa}$ indicated. (WT) Wild-type; $(t m 1 b) A 1 c f^{t m 1 b}$.

CRE-recombinase (Fig. 1A). Excision of this exon eliminates the coding sequence for the first AlCF RNA recognition motif, generates an in-frame premature stop codon, and a 
downstream frame-shift. This targeting strategy is designed to completely eliminate wild-type protein from the locus by generating a chimeric transcript encoding a short $\mathrm{N}$-terminal peptide from the endogenous locus and full-length $\beta$-galactosidase as a reporter. However, to our surprise, heterozygous $A 1 c f^{t m 1 b}$ crosses produced adult offspring in the expected Mendelian ratios $\left(n=57, \chi^{2}=0.579, P\right.$-value $\left.=0.749\right)$ in contrast to previously reported early embryonic lethality (Blanc et al. 2005). Given the conflict between our results and the previously published report, we wondered whether the A1c $f^{t m 1 b}$ allele was a true null allele. A1cf is highly expressed in the liver and small intestine (Dur et al. 2004), thus we tested whether homozygosity of the $A 1 c f^{t m 1 b}$ allele resulted in a loss of Alcf in these two tissues (Fig. 1B). qRT-PCR detection of the floxed exon and an exon boundary $5^{\prime}$ to the deleted exon confirmed exon 4 removal without the loss of $5^{\prime}$ junctions, as expected from the targeting strategy. To determine whether any mutant Alcf mRNA was generated from the locus, multiple exon junctions $3^{\prime}$ to the targeted exon were analyzed. This confirmed an almost complete loss of message in both liver and small intestine. To determine whether the mutant mRNA generated protein, Western blot analysis was used to detect A1CF in WT and mutant liver using a polyclonal antibody against the central portion of the protein. As expected, the dramatic reduction of A1cf message resulted in a complete loss of detectable protein (Fig. 1C). These findings demonstrate that the $A 1 c f^{t m 1 b}$ allele is a true null allele and does not result in embryonic lethality, in contrast to previous reports.

\section{A1CF loss does not impact C-to-U editing in the liver or small intestine}

A1CF has long been proposed as the major cofactor for APOBEC1, the only known catalytic component of the C-to-U editing complex. APOBEC1 loss results in a complete loss of $A p o B$ editing and given the viability of the $A 1 c f^{t m 1 b /}$ ${ }^{t m 1 b}$ mutants, we used $A 1 c f^{t m 1 b / t m 1 b}$ homozygous mice to assess whether global A1CF loss altered APOBEC1-dependent $\mathrm{C}$-to-U editing in the liver or small intestine, where A1CF is abundantly expressed (Sowden et al. 2004). Unexpectedly, Sanger sequencing of cDNA prepared from Alcf tmlb/tmlb homozygous mutants of the canonical APOBEC1 editing target, $A p o B$, suggested no substantial change in $A p o B$ C-to- $\mathrm{U}$ editing efficiency in either tissue (Fig. 2A). Given the nonquantitative nature of Sanger sequencing, we also utilized a previously reported qRT-PCR-based assay (Fossat et al. 2014) to quantify editing efficiency in WT and AlCF-null liver and small intestine. These analyses demonstrated no change in either editing efficiency or total $A p o B$ abundance with the loss of $\mathrm{A} 1 \mathrm{CF}$, indicating $\mathrm{APOBEC1}$ does not require A1CF to catalyze in vivo $A p o B$ editing in the liver and small intestine.

Previous reports have identified a number of other APOBEC1-dependent C-to-U editing events (Rosenberg
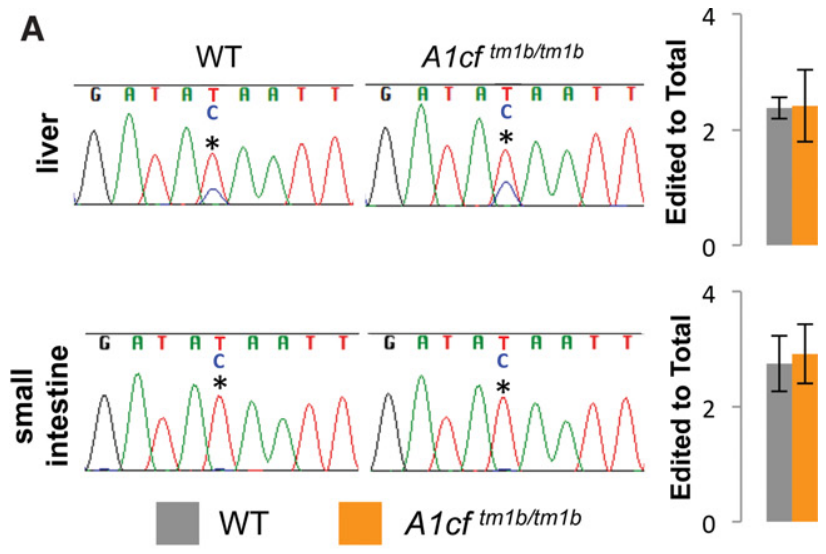

B Small Intestine editing
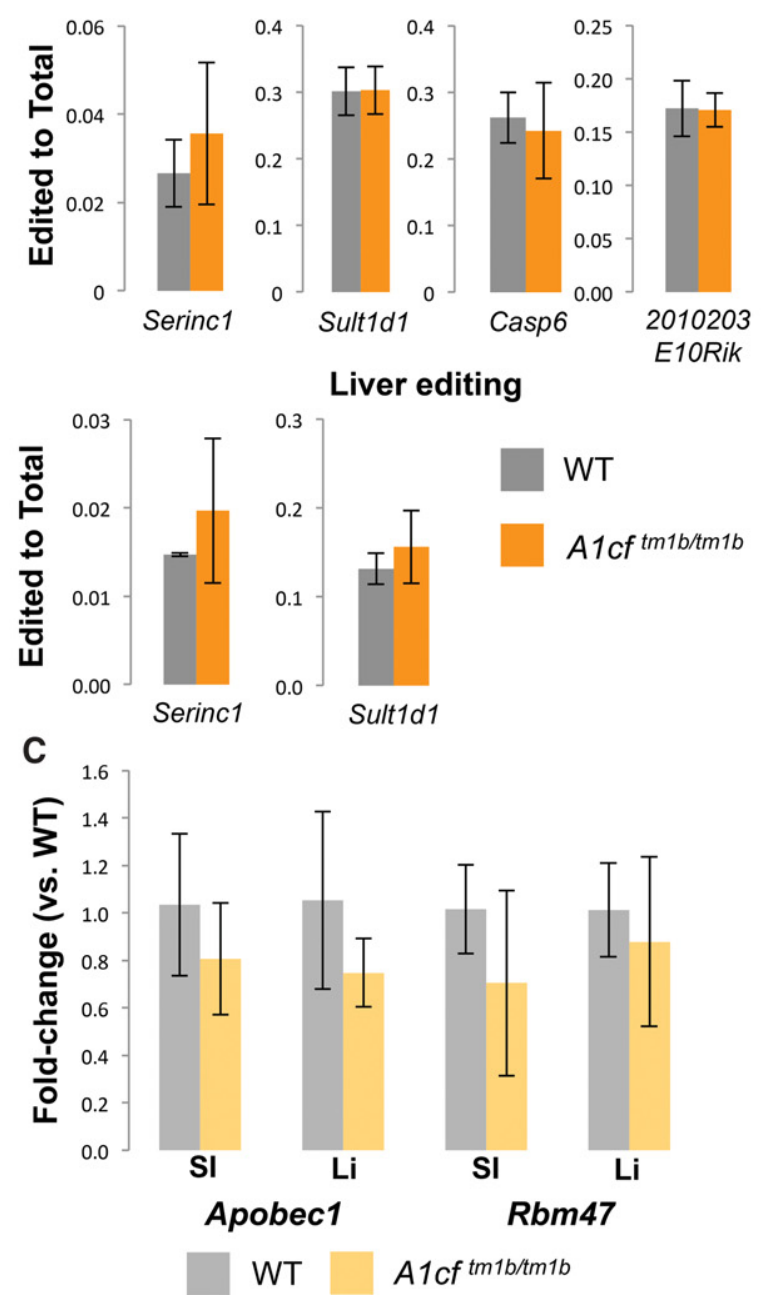

FIGURE 2. C-to-U editing is not impacted in the small intestine and liver with A1CF ablation. ( $A$ ) Sanger sequencing of $A p o B$ editing and qRT-PCR comparison of edited $A p o B$ (relative to total) in wild-type and homozygous $A 1 c f^{t m 1 b}$ small intestine and liver. (Asterisk) Editing site. (B) qRT-PCR comparison of multiple known editing sites in wild-type and homozygous $A 1 c f^{t m 1 b}$ small intestine and liver. $(C)$ qRT-PCR analysis of editing complex components in wild-type and homozygous $A 1 c f^{t m 1 b}$ small intestine and liver. $n \geq 4$, error, standard deviation. (WT) Wild-type; $(t m 1 b) A 1 c f^{t m 1 b}$; (SI) small intestine; (Li) liver. 
et al. 2011) that are lost with Apobecl mutation. Further supporting the notion that they are APOBEC1-dependent, all sites occur within stretches rich in AU sequence, similar to the known motif preference for APOBEC1 (MacGinnitie et al. 1995), and are accompanied by a downstream mooring sequence known to be required for APOBEC1 binding (Mehta and Driscoll 1998). We hypothesized that individual APOBEC1-dependent editing events may be relatively more or less sensitive to the loss of A1CF. To test whether this was the case, we compared editing efficiency across a number of previously confirmed APOBEC1-dependent RNA editing events in both the liver and the small intestine (Fig. 2B). As was the case for $A p o B$ editing, we detected no difference in the editing efficiency or total mRNA abundance in any of the known APOBEC1-dependent C-to-U editing targets we examined. Based on these observations, we conclude that A1CF is not required for APOBEC1-mediated C-to-U editing in either the liver or small intestine.

Gene compensation is a commonly observed phenomenon in gene ablation models (Rossi et al. 2015). Previous work has demonstrated RBM47 to be a necessary APOBEC1 cofactor (Fossat et al. 2014) and proposed a model whereby RBM47 interacted with both APOBEC1 and A1CF to form a functional editing complex. Given this model and the knowledge that RBM47 is expressed in both the liver and the small intestine, we wondered whether loss of A1CF was being compensated for by increases in either RBM47 or APOBEC1. To test this, we assessed both Apobec1 and Rbm47 expression in homozygous mutant liver and small intestine (Fig. 2C). No significant changes in expression were detected for either Apobec1 or Rbm47 with A1CF loss, demonstrating no significant transcriptional compensation for the loss of A1CF. From this evidence, we conclude normal C-to- $\mathrm{U}$ editing in A1CF mutants is not the result of $R m b 47$ transcriptional up-regulation.

\section{A1CF has novel physiological roles}

As global loss of A1CF did not result in embryonic lethality, we utilized the $A 1 c f^{t m 1 b}$ allele to determine the impact of A1CF loss on general mouse physiology. We analyzed phenotypic data generated by the JAX KOMP2 pipeline and available from the International Mouse Phenotyping Consortium (IMPC; www.mousephenotype.org/) and found significant impacts on the immune and hematopoietic system as well as altered homeostasis and metabolism. While no specific role for APOBEC1 has been reported in either the immune or hematopoietic system, knockouts do have altered cholesterol metabolism (Nakamuta et al. 1996).

Given our interest in understanding the in vivo role of A1CF as an APOBEC1 interacting factor and the known role of APOBEC1 in the mouse liver and small intestine, special attention was paid to metabolic measures associated with these tissues. Gross liver and small intestine morphology and the majority of phenotypic traits assessed showed no difference with A1CF loss. Of the analyzed traits, only the ratio of HDL to total cholesterol was significantly decreased in $A 1 c f^{t m 1 b}$ homozygous mutants ( $1.0544 \pm 0.0299$ versus $0.9600 \pm 0.0585, P$-value $=0.0185)$, driven primarily by increased total cholesterol $(66.6 \pm 4.2 \mathrm{mg} / \mathrm{dL}$ vs. $77.6 \pm 10.6$ $\mathrm{mg} / \mathrm{dL}, P$-value $=0.0811$ ), a finding observed only in male A1CF-null animals. This observation is in contrast to Apobec1 knockout mice, which display decreased HDL (Nakamuta et al. 1996).

A1CF has been reported by multiple groups to be highly expressed in the kidney (Lellek et al. 2000; Dur et al. 2004) and has been genetically (Pattaro et al. 2016) and molecularly (Huang et al. 2016) associated with kidney function. Given this, we evaluated whether our A1CF-null model displayed any kidney-related defects. Phenotypic analysis by the JAX KOMP2 program supported a potential role in kidney function as plasma analysis demonstrated increased protein and solute concentrations with A1CF loss (Fig. 3A), both of which indicate potential water homeostasis defects. As was the case for HDL concentrations, the impacts were more severe in males. Additionally, histological analysis of A1CFnull kidneys revealed a subtle but consistent abnormality of increased cellularity within glomeruli, again suggesting a potential water homeostasis defect in the knockout males (Fig. 3B). To examine the impact of A1CF on water homeostasis further, A1CF-null males were subjected to a multiday analysis of water intake and urine production. Over the course of the analysis, A1CF-null males showed no differences in water consumption or urine production when compared to wild-type controls. However, the concentration of both sodium and chloride was significantly decreased in A1CF-null urine as compared to wild-type (Fig. 3C). When taken in combination with the elevation of plasma solute concentration, we conclude the A1CF-null males have an underlying filtration defect, possibly due to abnormal cellularity in the kidney glomeruli.

\section{A1CF loss does not impact kidney RNA editing}

While A1CF loss did not appear to have any impact on APOBEC1-mediated RNA editing in either the liver or the small intestine, it remained a possibility that the A1CF-null kidney phenotype was due to its function as an APOBEC1 cofactor in that tissue. Quantitative RT-PCR of A1cf $f^{t m 1 b}$ adult kidney confirmed a similar pattern of $A 1 c f$ mRNA abundance as observed in both the liver and small intestine (Fig. 4A), similar to Apobec1 and Rbm47 expression as found in the liver, and minimal impact on Apobec1 and Rbm47 expression with A1CF loss (Fig. 4B). Taken together, we conclude that the impact of the $A 1 c f^{t m 1 b}$ allele on the expression of C-to$\mathrm{U}$ editing enzymes in the adult kidney is similar to what was observed in the liver and small intestine. We next asked whether C-to-U editing also occurred in the kidneys and whether it was impacted by A1CF loss. To that end, RNA sequencing of adult wild-type kidneys was examined for the 
A
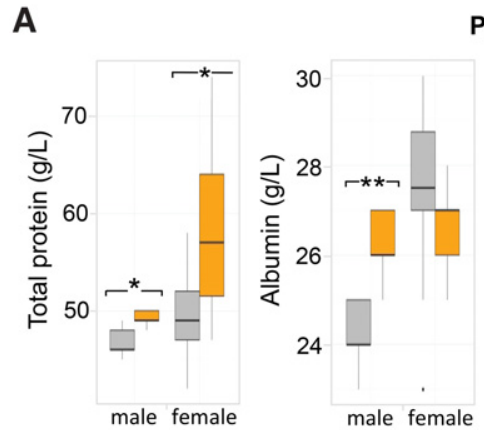

Plasma solutes

B

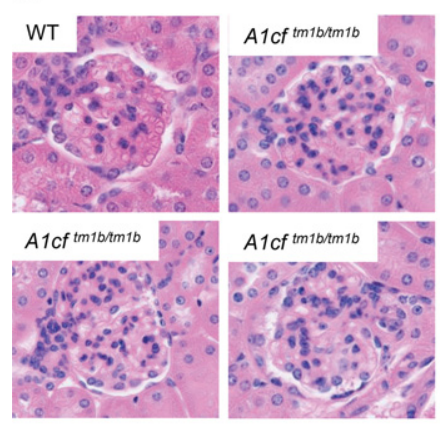

C
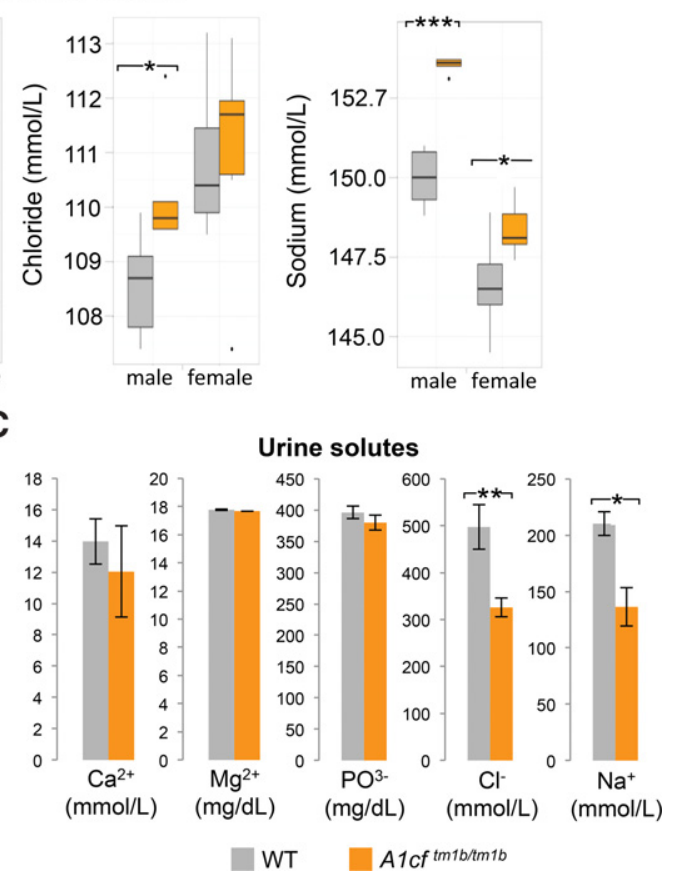

FIGURE 3. A1CF ablation impacts adult kidney physiology. (A) Plasma parameters in A1cf $f^{\mathrm{tm} 1 b}$ homozygous animals ( $n \geq 5$ per sex per genotype combination). (Asterisks) Significant differences ( $\left[{ }^{*}\right] P$-value $<0.05$, [ $\left.{ }^{* *}\right] P$-value $<0.01$, $\left[{ }^{* * *}\right] P$-value $\left.<0.0001\right)$. (B) Glomerular morphology in wild-type and homozygous $A 1 c f^{t m 1 b}$ kidney. $(C)$ Urine solute concentrations in A1cf $f^{\text {tm1b }}$ homozygous animals. $n=3$, error, standard deviation. $\left(\mathrm{Ca}^{2+}\right)$ Calcium; $\left(\mathrm{Mg}^{2+}\right)$ magnesium; $\left(\mathrm{PO}^{3-}\right)$ phosphate; $\left(\mathrm{Cl}^{-}\right)$chloride; $\left(\mathrm{Na}^{+}\right)$sodium; (WT) wild-type.

presence of C-to-U editing events using a custom RNA editing identification method (Fig. 4C and Materials and Methods). From this analysis, a set of high confidence Cto-U editing sites were defined and compared to a database of RNA editing sites (darned.ucc.ie/). While the majority of sites had not been previously observed, several had been detected in the liver ( $\mathrm{Gu}$ et al. 2012), the small intestine (Rosenberg et al. 2011), or both, suggesting that C-to-U editing of at least some targets is regulated by similar mechanisms across multiple tissues. Sanger sequencing was used to confirm kidney editing at selected sites prior to qRT-PCR analysis in the A1CF mutant kidneys (Fig. 4D). APOBEC1mediated editing sites are characterized by an AU-rich motif around the edited site as well as a downstream motif known as the mooring sequence. Motif analysis demonstrated that Cd36 contained both of these motifs in close association with the editing site, similar to other known APOBEC1-mediated editing events (Fig. 4E) and further validating the computational methods for $\mathrm{C}$-to- $\mathrm{U}$ editing site identification. As was found in the small intestine and liver, A1CF loss resulted in no significant alteration of either editing efficiency or target abundance in the kidney (Fig. 4F). From these findings, we conclude that while $\mathrm{A} 1 \mathrm{CF}$ is not required for APOBEC1-mediated C-to-U editing in vivo under normal physiological conditions, it is required for normal kidney physiology.

\section{DISCUSSION}

To date, the consensus within the literature has held that the primary function of $\mathrm{AlCF}$ is as a required co-factor for APOBEC1-mediated C-to-U editing. The results reported herein demonstrate $\mathrm{A} 1 \mathrm{CF}$ is not required for normal C-to$\mathrm{U}$ editing in the case of adult liver, small intestine, or kidney. However, the observation that global A1CF loss has an impact on normal kidney physiology indicates A1CF is playing some other function, presumably nonediting related, on which the kidney is particularly reliant. This report does not represent the first suggestion that AlCF may be important in nonediting-related RNA biology. In hepatic cells, A1CF has been shown to regulate both the stability (Blanc et al. 2010) and the subcellular localization of specific mRNAs (Galloway et al. 2010). Additional work demonstrated that $\mathrm{A} 1 \mathrm{CF}$ behaves as a nuclear shuttling protein (Blanc et al. 2003), and the shuttling is mediated by its interaction with APOBEC1 (Chester et al. 2003). These findings suggest a model whereby AlCF's primary role is to regulate the nuclear export of specific mRNA species. There is also evidence to suggest that A1CF itself is sensitive to metabolic changes (Sowden et al. 2002, 2004; Blanc et al. 2003) or disease state (Galloway et al. 2010), and it remains an open question whether altered $\mathrm{A} 1 \mathrm{CF}$ changes affect $\mathrm{C}$-to- $\mathrm{U}$ editing in those kinds of environments.

The lack of changes in APOBEC1-mediated editing with $\mathrm{A} 1 \mathrm{CF}$ loss was unexpected given the abundance of in vitro evidence demonstrating $\mathrm{A} 1 \mathrm{CF}$ interacts with and complements APOBEC1 editing activity. However, while in vitro and overexpression studies define a protein's capacity for a specific molecular function, genetic in vivo analyses are necessary to demonstrate strict reliance on that protein for a specific molecular event. Given the lack of editing changes with global A1CF loss, it appears as though while A1CF is capable of complementing APOBEC1-mediated editing in vitro, $\mathrm{A} 1 \mathrm{CF}$ is not required for that function in vivo. This conclusion is supported by a failure to find genetic variation in C-to- $\mathrm{U}$ editing linked to either $\mathrm{AlCF}$, or the mooring sequence for $\mathrm{A} 1 \mathrm{CF}$, in a genetically diverse multiparent mouse population (Gu et al. 2016). Altered editing efficiencies at the $A p o B$ locus and 49 other $\mathrm{C}$-to- $\mathrm{U}$ edited sites appeared to be solely driven by linkage to four functional Apobecl alleles segregating within the population. Additionally, SNPs within the mooring sequence or small indels between the edited site and the mooring sequence did not affect the editing efficiency of 


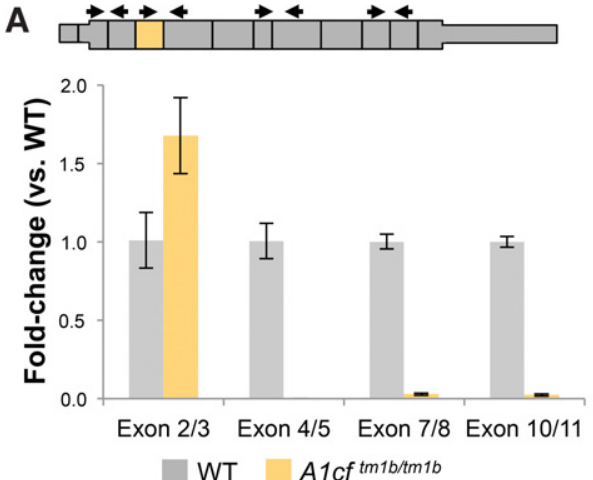

C

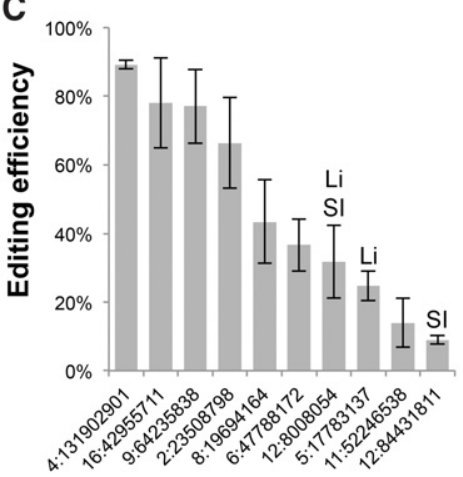

D

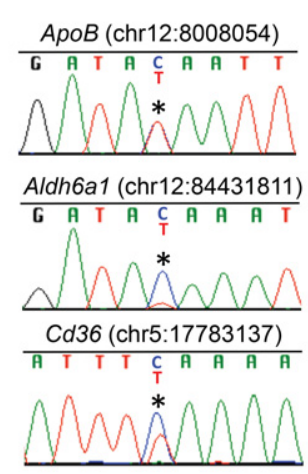

$\mathbf{F}$

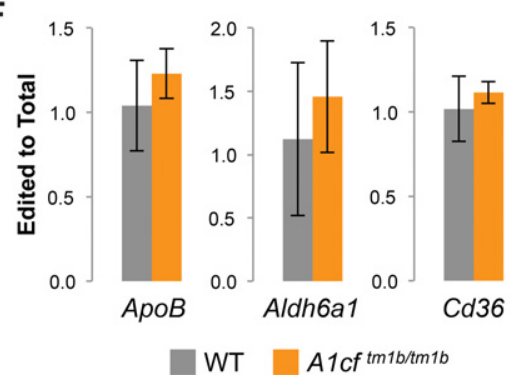

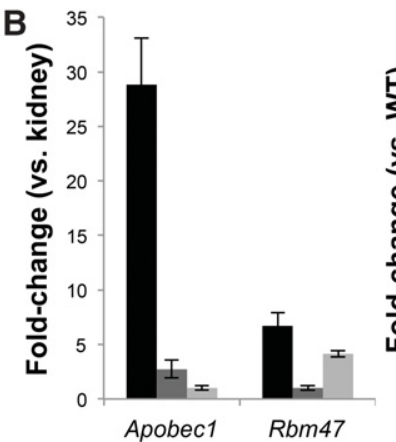

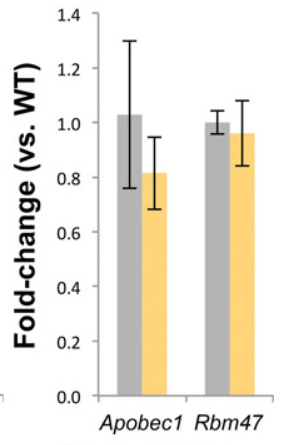

E

\section{$\underline{A p o B}(\mathrm{chr12:8008054)}$} gcGaua Canuugaucaguauau

Aldh6a1 (chr12:84431811)

CAgaua CaAauagauccuuagau

Cd36 (chr5:17783137)

auduuUCAaAagauUaAuauguc

FIGURE 4. Kidney C-to-U editing occurs in a distinct set of targets and is not impacted with A1CF ablation. (A) Alcf mRNA expression in WT and $A 1 c f^{t m 1 b}$ adult kidney assessed by qRT-PCR. Exon junctions indicated in graph and on WT mRNA schematic above ( $n \geq 4$, error, standard deviation). (B) Expression of known C-to-U editing enzymes in WT and Alcf $f^{t m 1 b}$ adult kidney as compared to small intestine and liver $(n \geq 4$, error, standard deviation). (C) Editing efficiency at computationally defined C-to-U editing sites in the adult kidney including site overlap with known sites in the small intestine (SI) and liver (Li). $n=3$, error, standard deviation. (D) Sanger sequencing confirmation of select editing sites in adult kidney. (Asterisk) Editing site. (E) APOBEC1 recognition site (yellow) and mooring sequence (blue) motif identification within known APOBEC1-dependent (Apob and Aldh6a1) and newly identified (Cd36) editing sites. (F) qRT-PCR comparison of edited transcript (relative to total) of select C-to-U editing sites in wild-type and homozygous $A 1 c f^{t m 1 b}$ adult kidney $n=4$, error, standard deviation. (WT) Wild-type.

the RNA. It is possible other APOBEC enzymes are compensating for lack of APOBEC1 editing activity in A1CF mutants, as recent work has demonstrated human APOBEC3A is capable of catalyzing C-to-U editing in monocytes and macrophages (Sharma et al. 2015). However, this possibility seems unlikely as mouse APOBEC3 is not known to have editing activity and is only distantly related to human APOBEC3A, which has a site recognition sequence distinct from that of APOBEC1 and is not found in any of the targets examined in this work.

RBM47 has been demonstrated to be required for normal C-to-U editing in vivo as RBM47 mutants have a near com- plete loss of RNA editing (Fossat et al. 2014), in contrast to what was observed herein as well as in a previously reported A1CF mutant allele (Blanc et al. 2005). Additionally, APOBEC1-mediated editing in both cell and cell-free editing assays is complemented by RBM47, an activity not further enhanced by the addition of A1CF. In combination, our genetic and molecular observations in the context of A1CF loss and the observations of Fossat and coworkers suggest a model whereby RBM47 acts alone as the in vivo APOBEC1 complementation factor. Further experimental validation will be required to exhaustively test this model. It should be noted, this model does not exclude the possibility that A1CF plays 
some other role in modulating APOBEC1 editing activity. Although not significant, many of the editing sites examined in the context of the $A 1 c f^{t m 1 b}$ allele showed a moderate increase in editing efficiency relative to wild-type. This finding is somewhat reminiscent of the increase in editing efficiency observed in heterozygotes carrying the previously reported A1cf knockout allele $\left(A 1 c f^{t m 1 D d s n}\right)$. Unfortunately, RNA editing has not been assessed in the only other known A1cf ablation model (Harkins and Whitton 2016).

Direct comparison of the various Alcf knockout alleles is complicated by the difference in the phenotypes they generate. Failing genome-wide, side-by-side comparison, it may be difficult to determine the exact cause of the differences in phenotype between the two viable global A1CF ablation models and the A1cf $f^{t m 1 D d s n}$ allele. However, multiple lines of evidence suggest the $A 1 c f^{t m 1 D d s n}$ allele may have additional undetected genetic aberrations. Firstly, the embryonic lethality reported for $A 1 c f^{t m 1 D d s n}$ occurs early in gestation, between E3.5 and E7.5, and prior to the reported onset of A1cf expression (Blanc et al. 2005). Secondly, a recent report demonstrated that the Alcf $f^{\text {mIDdsn }}$ allele displays a transmission distortion ratio (Carouge et al. 2016), which is often indicative of chromosomal rearrangements such as Robertsonian translocations (Underkoffler et al. 2005). Taken together, this evidence suggests the A1cf $f^{t m 1 D d s n}$ allele phenotype may be a result of additional, unintended genetic alterations unrelated to the loss of A1CF. Given our confirmation of A1CF ablation at the DNA, mRNA, and protein level in multiple adult tissues, we are confident the $A 1 c f$ allele reported herein genuinely represents the phenotypic impact of A1CF ablation. While it is possible that both alleles represent true A1CF global nulls, it seems more reasonable that unexpected genomic impacts or genetic interactions have led to a more extreme phenotype in the previously reported model.

\section{MATERIALS AND METHODS}

\section{Production and maintenance of the $A 1 c f^{f m 1 b}$ allele}

All animal work used in this study was approved by The Jackson Laboratory Animal Care and Use Committee (Permit Number: 07007) and are in accordance with the "Guide for the Care and Use of Experimental Animals" established by the National Institutes of Health (1996, revised 2011). Animals were maintained in a 12-h light and 12-h dark cycle vivarium and provided water and autoclaved pelleted $5 \mathrm{~K} 52$ diet ( $6 \%$ fat) ad libitum. Animals carrying

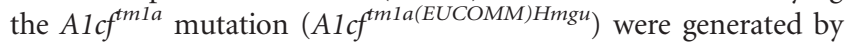
the Jackson Lab KOMP2 program. Details of its generation and structure are provided at http://jaxmice.jax.org/strain/024325.html. Alcf $f^{t m 1 a}$ mice were crossed to Sox2-Cre carrying mice (https:// www.jax.org/strain/014094) to generate $A 1 c f^{t m 1 b}$ offspring, which were then intercrossed to produce homozygous $A 1 c f^{t m 1 b}$ experimental animals. All experimental and control animals were maintained on a C57BL/6NJ background. Genotyping was performed using the following primers and conditions: Common_F (CCCA GGCCACCTATGAAATA) and Common_R (TCTTACCCCTCC
TCGGTTTT) to generate the wild-type product and Common_F and Alcf_AF (GTTGTTAACTTGTTTATTGC) to generate the $A 1 c f^{t m 1 b}$-specific product with the following cycling conditions: $94^{\circ} \mathrm{C}$ for $5 \mathrm{~min} ; 94^{\circ} \mathrm{C}$ for $30 \mathrm{sec}, 55.9^{\circ} \mathrm{C}$ for $30 \mathrm{sec}, 72^{\circ} \mathrm{C}$ for $1 \mathrm{~min}$ (30 times); $72^{\circ} \mathrm{C}$ for $5 \mathrm{~min}$.

\section{RNA isolation, reverse transcription, total template quantitative RT-PCR, and Sanger sequencing template production}

RNA was isolated from wild-type and $A 1 c f^{t m 1 b}$ small intestine, liver, and kidney using TRIzol reagent (ThermoFisher) following manufacturer recommended methods. Samples were DNase treated (RNAse free DNAse set, Qiagen) and cDNA generated using the Superscript III First-Strand Synthesis System for RT-PCR (Life Technologies) with random hexamers and following manufacturer recommended conditions. Quantitative analysis of cDNA abundance was performed using SYBR Green PCR Master Mix (Applied Biosystems) amplification on a 7500 Real Time PCR System instrument (Applied BioSystems). The following primer pairs were used for total gene abundance analysis by traditional quantitative RT-PCR: Alcf exon 2/3 (F-TCCAGCGCACAGG ATATAGC, R-TGAAAATCTCGCAGCCCCTT), Alcf exon 4/5 (F-CAGGAAGCCAAGAATGCAATCA, R-TCCTCCCACAAAC AATCGGC), A1cf exon 7/8 (F-CCGAGACTACGCTTTTGTGC, R-ATGGCTCAGAGGGTAGGTGT), Alcf exon 10/11 (F-TCT GCCATTGGACAAGATCA, R-GCGCTTAGCTTTGGTGGTAT), Apobec1 (F-TACATAGCACGGCTTTATCACCAC, R-AGTCAC ACCGCTGCTAATAAGGTC), and Rbm47 (F-GTCATTCCTGC GGTATCCACAC, R-CTGAACATTTGGTGCCACGG). Total gene abundance is reported as relative to the endogenous control Rps2 (F-CTGACTCCCGACCTCTGGAAA， R-GAGCCTGGGTCCT CTGAACA). Templates for Sanger sequencing were generated using the following template-specific primer pairs: Apob (FCAAGTAGCTGGTGCCAAGGA, R-TTTGTGTCCTGAGCTGC TGT), Aldh6a1 (F-GTTGAGCCTCAAATGCAGCC, R-AGAAG CAAGCTTAAAGGCAGC), and Cd36 (F-GGTGGTGTGTGCTC TCTCTC, R-GCTGACAGTTGCAAGCCAAA). Sequencing products were visualized using Sequencher v 5.1 (Gene Codes).

\section{Quantitative RT-PCR comparison of editing versus nonediting targets}

SYBR Green PCR Master Mix (Applied Biosystems) amplification of total and edited templates was analyzed using a 7500 Real-Time PCR System instrument (Applied BioSystems) and total versus edited ratios compared. Primers for the following targets were as described in Fossat et al. (2014): Apob, Serinc1, Sult1d1, Casp6, and 20102302E10Rik. Using the previously described method additional primers were designed for the following targets: Aldh6a1 (F-GTT GAGCCTCAAATGCAGCC with either R1-GGGAGATCCTTT GATTTCTGGGT to detect total abundance, or R2-GATTTTA TCTAAGGATCTATTTA to detect edited abundance) and Cd36 (F-GGTGGTGTGTGCTCTCTCTC with either R1-GCTGACA GTTGCAAGCCAAA to detect total abundance, or R2-GTGACA TATTAATCTTTTA to detect edited abundance). In all cases, relative abundance was calculated via the ddCt method with Rps2 as an endogenous control. 


\section{Protein isolation and immunodetection of $\mathrm{A1CF}$}

Total protein was isolated from small intestine, liver, and kidney by flash freezing followed by pulverization into fine powder on dry ice using a mortar and pestle. Powders were dissolved in SDS loading buffer minus dithiothreitol (100 mM Tris-Cl, pH 6.8; 4\% SDS, $0.2 \%$ bromophenol blue, $20 \%$ glycerol) and boiled after the addition of $200 \mathrm{mM}$ dithiothreitol followed by electrophoresis on a $10 \%$ SDS-polyacrylamide gel. Following electrophoresis, proteins were transferred to an Immobilon PVDF membrane (Millipore) and the membrane blocked for an hour in 5\% nonfat dry milk in Tris-buffered saline (PBS). Primary antibodies (Atlas anti-A1CF, HPA044079, 1:500 or Abcam anti-GAPDH, 1:2500) incubation occurred overnight at $4^{\circ} \mathrm{C}$, followed by washing in PBS with $0.1 \%$ Tween-20 and secondary antibody (BioRad anti-rabbit HRP, 172$1019,1: 5000)$ incubation. Following incubation, the membrane was washed with PBS with $0.1 \%$ Tween-20 and developed using SuperSignal West Pico Chemiluminescent Substrate (Thermo Scientific) followed by imaging using a G:Box Chemi XT4 (Syngene).

\section{Phenotypic analyses}

Clinical plasma chemistry and body composition phenotypic data for $A 1 c f^{t m 1 b}$ mutant mice were generated by the JAX KOMP2 Phenotyping pipeline. Phenotyping details and data are available from the International Knockout Mouse Consortium (IMPC). Wild-type and mutant data points were matched based on animal date of birth and assay date. Statistical analyses were performed using JMP 11. Tissue collection, embedding, sectioning, and hematoxylin/eosin staining for histological evaluation were performed by Histological Services at The Jackson Laboratory. Detailed histological evaluation was performed by a board certified staff pathologist on at least two adult samples of each sex for each genotype. Additional metabolic parameters (urine analysis, water and food consumption, detailed weight loss, and gain) were collected for adult (42-65 d post-partum) wild-type and $A 1 c f^{t m 1 b}$ mice using Metabolic Cages for Single Mouse (Techniplast) over a 5-d period. Urine solute concentrations were assessed on a Beckman Coulter AU600 System by xylidyl blue (magnesium), molybdate (phosphate), arsenazo (calcium), a modified version of the Jaffe procedure (creatinine), turbidimeters (albumin), or the ISE module (sodium and chloride).

\section{In silico editing analysis}

Total male C57BL6N/J adult kidney RNA was isolated via RNeasy Mini Column (Qiagen) purification. Sequencing libraries were constructed using the Stranded Total RNA LT with Ribo-Zero TM Gold Library Prep kit (Illumina) and paired-end 100-bp reads sequenced on an Illumina HiSeq 2500 to a minimum depth of 30 million reads per sample. Computational editing identification was based on the protocol described in Ramaswami et al. (2013). In brief, quality and duplicate filtered reads were trimmed to remove random hexamer sequence and aligned to the C57BL6N/J genome via Tophat. Variants were called using the GATK UnifiedGenotyper and filtered for minimum base and mapping quality. Output sites were further filtered to those residing within exons, represented by only a single variant type, supported by at least 10 reads, occurring in $>5 \%$ and $<95 \%$ of total reads, and observed in all three biological replicates.
Of these, variants representing C-to-T events were reported as putative C-to-U editing sites.

\section{ACKNOWLEDGMENTS}

The authors thank Dr. Rosalinda Doty, DMV for kidney histological evaluation, Dr. Dayana Krawchuk for figure preparation, and the Clinical Assessment Laboratory of the Jackson Laboratory Histopathology services for technical support. Funding support was provided by National Human Genome Research Institute (NHGRI) grant HG006332, National Institute of Child Health and Human Development (NICHD) grant HD083521, and National Institutes of Health (NIH), Office of the Director, 5U54OD020351-02.

Received August 19, 2016; accepted December 14, 2016.

\section{REFERENCES}

Blanc V, Kennedy S, Davidson NO. 2003. A novel nuclear localization signal in the auxiliary domain of apobec-1 complementation factor regulates nucleocytoplasmic import and shuttling. J Biol Chem 278: $41198-41204$.

Blanc V, Henderson JO, Newberry EP, Kennedy S, Luo J, Davidson NO. 2005. Targeted deletion of the murine apobec-1 complementation factor (acf) gene results in embryonic lethality. Mol Cell Biol 25: $7260-7269$.

Blanc V, Sessa KJ, Kennedy S, Luo J, Davidson NO. 2010. Apobec-1 complementation factor modulates liver regeneration by post-transcriptional regulation of interleukin-6 mRNA stability. J Biol Chem 285: 19184-19192.

Carouge D, Blanc V, Knoblaugh SE, Hunter RJ, Davidson NO, Nadeau JH. 2016. Parent-of-origin effects of AlCF and $\mathrm{AGO}_{2}$ on testicular germ-cell tumors, testicular abnormalities, and fertilization bias. Proc Natl Acad Sci 113: E5425-E5433.

Chester A, Somasekaram A, Tzimina M, Jarmuz A, Gisbourne J, O'Keefe R, Scott J, Navaratnam N. 2003. The apolipoprotein B mRNA editing complex performs a multifunctional cycle and suppresses nonsense-mediated decay. EMBO J 22: 3971-3982.

Chester A, Weinreb V, Carter CW Jr, Navaratnam N. 2004. Optimization of apolipoprotein B mRNA editing by APOBEC1 apoenzyme and the role of its auxiliary factor, ACF. RNA (New York, NY) 10: 1399-1411.

Davies MS, Wallis SC, Driscoll DM, Wynne JK, Williams GW, Powell LM, Scott J. 1989. Sequence requirements for apolipoprotein B RNA editing in transfected rat hepatoma cells. J Biol Chem 264: 13395-13398.

Driscoll DM, Casanova E. 1990. Characterization of the apolipoprotein B mRNA editing activity in enterocyte extracts. J Biol Chem 265: 21401-21403.

Driscoll DM, Wynne JK, Wallis SC, Scott J. 1989. An in vitro system for the editing of apolipoprotein B mRNA. Cell 58: 519-525.

Dur S, Krause K, Pluntke N, Greeve J. 2004. Gene structure and expression of the mouse APOBEC-1 complementation factor: multiple transcriptional initiation sites and a spliced variant with a premature stop translation codon. Biochim Biophys Acta 1680: 11-23.

Fossat N, Tourle K, Radziewic T, Barratt K, Liebhold D, Studdert JB, Power M, Jones V, Loebel DA, Tam PP. 2014. C to U RNA editing mediated by APOBEC1 requires RNA-binding protein RBM47. EMBO Rep 15: 903-910.

Galloway CA, Ashton J, Sparks JD, Mooney RA, Smith HC. 2010. Metabolic regulation of APOBEC-1 complementation factor trafficking in mouse models of obesity and its positive correlation with the expression of $\mathrm{ApoB}$ protein in hepatocytes. Biochim Biophys Acta 1802: 976-985. 
Gu T, Buaas FW, Simons AK, Ackert-Bicknell CL, Braun RE, Hibbs MA. 2012. Canonical A-to-I and C-to-U RNA editing is enriched at $3^{\prime}$ UTRs and microRNA target sites in multiple mouse tissues. PLoS One 7: e33720.

Gu T, Gatti DM, Srivastava A, Snyder EM, Raghupathy N, Simecek P, Svenson KL, Dotu I, Chuang JH, Keller MP, et al. 2016. Genetic architectures of quantitative variation in RNA editing pathways. Genetics 202: 787-798.

Harkins S, Whitton JL. 2016. Chromosomal mapping of the aMHCMerCreMer transgene in mice reveals a large genomic deletion. Transgenic Res 25: 639-648.

Hartner JC, Schmittwolf C, Kispert A, Muller AM, Higuchi M, Seeburg PH. 2004. Liver disintegration in the mouse embryo caused by deficiency in the RNA-editing enzyme ADAR1. J Biol Chem 279: 4894-4902.

Higuchi M, Maas S, Single FN, Hartner J, Rozov A, Burnashev N, Feldmeyer D, Sprengel R, Seeburg PH. 2000. Point mutation in an AMPA receptor gene rescues lethality in mice deficient in the RNA-editing enzyme ADAR2. Nature 406: 78-81.

Hirano K, Young SG, Farese RV Jr, Ng J, Sande E, Warburton C, PowellBraxton LM, Davidson NO. 1996. Targeted disruption of the mouse apobec-1 gene abolishes apolipoprotein B mRNA editing and eliminates apolipoprotein B48. J Biol Chem 271: 9887-9890.

Huang L, Wang H, Zhou Y, Ni D, Hu Y, Long Y, Liu J, Peng R, Zhou L, Liu Z, et al. 2016. Apobec-1 complementation factor (A1CF) inhibits epithelial-mesenchymal transition and migration of normal rat kidney proximal tubular epithelial cells. Int J Mol Sci 17: 197.

Lellek H, Kirsten R, Diehl I, Apostel F, Buck F, Greeve J. 2000. Purification and molecular cloning of a novel essential component of the apolipoprotein B mRNA editing enzyme-complex. J Biol Chem 275: 19848-19856.

MacGinnitie AJ, Anant S, Davidson NO. 1995. Mutagenesis of apobec1 , the catalytic subunit of the mammalian apolipoprotein B mRNA editing enzyme, reveals distinct domains that mediate cytosine nucleoside deaminase, RNA binding, and RNA editing activity. J Biol Chem 270: 14768-14775.

Mehta A, Driscoll DM. 1998. A sequence-specific RNA-binding protein complements apobec-1 to edit apolipoprotein B mRNA. Mol Cell Biol 18: 4426-4432.

Mehta A, Driscoll DM. 2002. Identification of domains in apobec-1 complementation factor required for RNA binding and apolipoprotein-B mRNA editing. RNA 8: 69-82.

Mehta A, Kinter MT, Sherman NE, Driscoll DM. 2000. Molecular cloning of apobec-1 complementation factor, a novel RNA-binding protein involved in the editing of apolipoprotein B mRNA. Mol Cell Biol 20: $1846-1854$.

Nakamuta M, Oka K, Krushkal J, Kobayashi K, Yamamoto M, Li WH, Chan L. 1995. Alternative mRNA splicing and differential promoter utilization determine tissue-specific expression of the apolipoprotein B mRNA-editing protein (Apobec1) gene in mice. Structure and evolution of Apobec1 and related nucleoside/nucleotide deaminases. J Biol Chem 270: 13042-13056.

Nakamuta M, Chang BH, Zsigmond E, Kobayashi K, Lei H, Ishida BY, Oka K, Li E, Chan L. 1996. Complete phenotypic characterization of apobec-1 knockout mice with a wild-type genetic background and a human apolipoprotein B transgenic background, and restoration of apolipoprotein B mRNA editing by somatic gene transfer of Apobec1. J Biol Chem 271: 25981-25988.

Pattaro C, Teumer A, Gorski M, Chu AY, Li M, Mijatovic V, Garnaas M, Tin A, Sorice R, Li Y, et al. 2016. Genetic associations at 53 loci highlight cell types and biological pathways relevant for kidney function. Nat Commun 7: 10023.

Powell LM, Wallis SC, Pease RJ, Edwards YH, Knott TJ, Scott J. 1987. A novel form of tissue-specific RNA processing produces apolipoprotein-B48 in intestine. Cell 50: 831-840.

Ramaswami G, Zhang R, Piskol R, Keegan LP, Deng P, O'Connell MA, Li JB. 2013. Identifying RNA editing sites using RNA sequencing data alone. Nat Methods 10: 128-132.

Rosenberg BR, Hamilton CE, Mwangi MM, Dewell S, Papavasiliou FN. 2011. Transcriptome-wide sequencing reveals numerous APOBEC1 mRNA-editing targets in transcript 3' UTRs. Nat Struct Mol Biol 18: 230-236.

Rossi A, Kontarakis Z, Gerri C, Nolte H, Holper S, Kruger M, Stainier DY. 2015. Genetic compensation induced by deleterious mutations but not gene knockdowns. Nature 524: 230-233.

Severi F, Conticello SG. 2015. Flow-cytometric visualization of $\mathrm{C}>\mathrm{U}$ mRNA editing reveals the dynamics of the process in live cells. RNA Biol 12: 389-397.

Sharma S, Patnaik SK, Taggart RT, Kannisto ED, Enriquez SM, Gollnick P, Baysal BE. 2015. APOBEC3A cytidine deaminase induces RNA editing in monocytes and macrophages. Nat Commun 6: 6881.

Smith HC, Kuo SR, Backus JW, Harris SG, Sparks CE, Sparks JD. 1991. In vitro apolipoprotein $B$ mRNA editing: identification of a $27 \mathrm{~S}$ editing complex. Proc Natl Acad Sci 88: 1489-1493.

Sowden MP, Ballatori N, Jensen KL, Reed LH, Smith HC. 2002. The editosome for cytidine to uridine mRNA editing has a native complexity of 27S: identification of intracellular domains containing active and inactive editing factors. J Cell Sci 115: 1027-1039.

Sowden MP, Lehmann DM, Lin X, Smith CO, Smith HC. 2004 Identification of novel alternative splice variants of APOBEC-1 complementation factor with different capacities to support apolipoprotein B mRNA editing. J Biol Chem 279: 197-206.

Teng B, Verp M, Salomon J, Davidson NO. 1990. Apolipoprotein B messenger RNA editing is developmentally regulated and widely expressed in human tissues. J Biol Chem 265: 20616-20620.

Teng B, Burant CF, Davidson NO. 1993. Molecular cloning of an apolipoprotein B messenger RNA editing protein. Science 260: 1816-1819.

Underkoffler LA, Mitchell LE, Abdulali ZS, Collins JN, Oakey RJ. 2005. Transmission ratio distortion in offspring of mouse heterozygous carriers of a (7.18) Robertsonian translocation. Genetics 169: 843-848. 

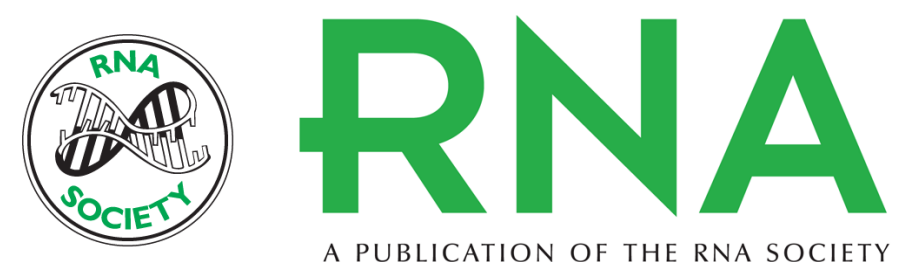

A PUBLICATION OF THE RNA SOCIETY

\section{APOBEC1 complementation factor (A1CF) is dispensable for C-to-U RNA editing in vivo}

Elizabeth M. Snyder, Christopher McCarty, Adrienne Mehalow, et al.

RNA 2017 23: 457-465 originally published online January 9, 2017

Access the most recent version at doi:10.1261/rna.058818.116

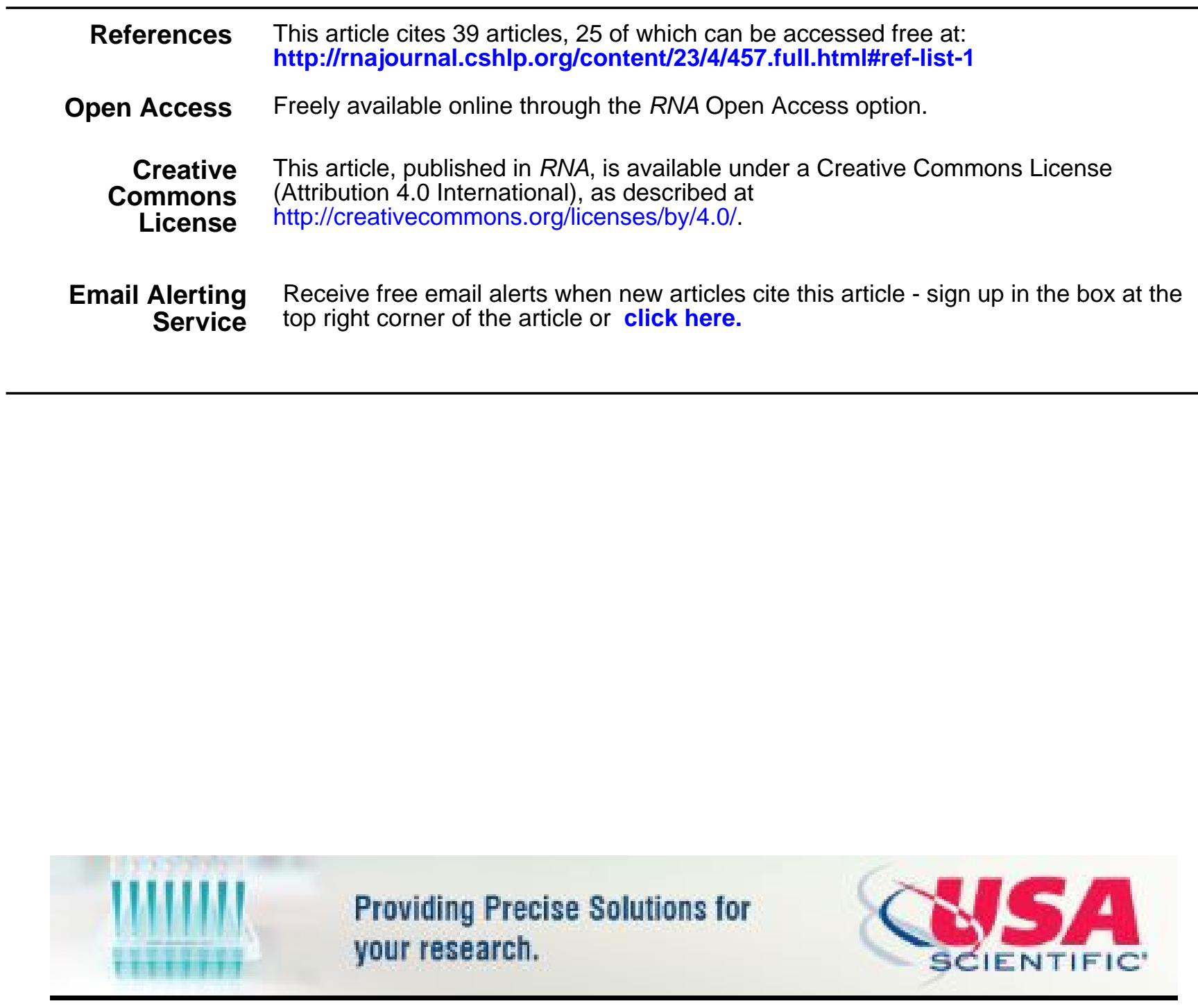

To subscribe to $R N A$ go to:

http://rnajournal.cshlp.org/subscriptions

(C) 2017 Snyder et al.; Published by Cold Spring Harbor Laboratory Press for the RNA Society 